\title{
FREQÜÊNCIA AO AMBIENTE HUMANO E DISPERSÃO DE MOSQUITOS CULICIDAE EM ÁREA ADJACENTE À MATA ATLÂNTICA PRIMITIVA DA PLANÍCIE*
}

\author{
Oswaldo Paulo Forattini** \\ Almério de Castro Gomes** \\ Jair Lício Ferreira Santos** \\ Iná Kakitani** \\ Daniel Marucci**
}

FORATTINI, O. P. et al. Freqüência ao ambiente humano e dispersão de mosquitos Culicidae em área adjacente à mata atlântica primitiva da planície. Rev. Saúde públ., S. Paulo, 24: 101-7, 1990.

RESUMO: Apresentam-se resultados obtidos comł observações em área adjacente a ambiente primitivo representado pela mata atlântica da planície. Focalizam-se as espécies Aedes scapularis, Anopheles bellator, An. cruzii e Culex sacchettae. A dispersão foi estudada com a utilização de hospedeiro único e que assim permitiu o reconhecimento dos mosquitos pela identificacão do sangue ingerido. A freqüência ao ambiente humano foi observada mediante a realização de coletas com aspiração e utilização de isca humana, no peridomicílio e na mata circunjacente. Ae. scapularis revelou acentuada capacidade de dispersão apetente, bem como permanência no ambiente domiciliar. Os anofelinos Kerteszia mostraram tendência ao abandono desse meio e capacidade de retorno pós-prandial ao ambiente florestal primitivo. $\mathrm{O} \mathrm{Cx}$. sacchettae, juntamente com as outras três espécies, revelou freqüência regular ' $a$ isca humana no peridomicilio. A estimativa do índice alimentar ("feeding index") para Ae. scapularis mostrou preferência por animais de grande porte, principalmente bovinos. Considera-se que no mecanismo de domiciliação, desses mosquitos, a presença de fontes sangüíneas, representada por número significante de animais domésticos de grande porte, constitui fator de atração para populaçôes de mosquitos silvestres existentes na mata adjacente. Destes, aqueles que tendem a permanecer no ambiente humano, após a realização do repasto sanguíneo, assumem particular significado na transmissão de agentes infecciosos, destacando Ae. scapularis e $\mathrm{C}$. sacchettae.

DESCRITORES: Mosquitos. Aedes scapularis. Anopheles. Culex sacchettae. Dispersão populacional. Domiciliação. Preferências alimentares.

\section{INTRODUÇÃO}

As observações sobre o comportamento de mosquitos Culicidae no Vale do Ribeira, Estado de São Paulo, Brasil, têm destacado significado epidemiológico, reconhecido ou potencial, de Aedes scapularis e de alguns representantes de Culex (Melanoconion), além de anofelinos do subgênero Kerteszia. Outros culicídeos foram objeto desses estudos, em que pese até o momento não terem revelado traço comportamental mais significativo, no sentido de possível incriminação vetora (Forattini e col. ${ }^{5,6,7}$ 1987, 1989). Diante disso, julgou-se oportuno levar a efeito nova série de observações, com a finalidade de focalizar particularmente aquelas espécies.

O contato entre mosquito e possível vertebrado está sujeito à influência de fatores múltiplos como, além de outros, a complexa interação entre a preferência alimentar, as tentativas de sugar, a realimentação e a reação comportamental do hospedeiro (DeFoliart e col. ${ }^{3}$, 1987). Nesse particular, pode-se encontrar diferenças substanciais na atividade de adultos, mesmo em espécies proximamente relacionadas. Assim sendo, no presente trabalho procurou-se focalizar alguns daqueles aspectos em ambiente antropicamente alterado, mas adjacente a meio primitivo, preservado ao menos em suas linhas gerais.

Nesta abordagem, e no caso das formas adultas, pretende-se obter dados que, apesar de próprios à localidade em estudo, pudessem fornecer informações não apenas da freqüência aos domicílios, mas também sobre a mobilidade resultante da dispersão apetente (Johnson ${ }^{10}, 1969$; Ginsberg $\left.{ }^{9}, 1986\right)$. São relativamente escassos os dados disponíveis sobre a movimentação pós-prandial de culicídeos. Algumas observações foram realizadas com anofelinos, como Anopheles gambiae s.s. em Gambia e Nigéria. Em vilarejos dessas regiões pôde-se evidenciar a mobilidade, de casa para casa, de fêmeas alimentadas (Boreham e col. ${ }^{1}, 1979$; Boreham e Port ${ }^{2}$, 1982). Esse comportamento reveste-se de as-

\footnotetext{
* Realizado com auxílio financeiro da Fundação de Amparo à Pesquisa do Estado de S. Paulo - FAPESP. (Processo $n^{2} 86 / 3195-8$ ).

* Departamento de Epidemiologia da Faculdade de Saúde Pública da Universidade de São Paulo - Av. Dr. Arnaldo, 715 - 01255 - São Paulo, SP - Brasil.
} 
pecto significativo para o mecanismo de transmissão da malária naquelas regióes. Em nosso meio existe carência desse tipo de observações, o que pelo menos, em parte, se justifica face ao grau de domiciliação consideravelmente menor que apresentam nossas populaçðes vetoras. Todavia, julgou-se oportuna a realização de pesquisas sobre esse comportamento, por parte de algumas espécies encontradas em área rural e, portanto, artificialmente instalada pelo homem. Ao contrário dos exemplos supracitados, esse meio caracteriza-se pelo pequeno número de habitaçס̃es, esparsamente situadas sobre o terreno modificado mas circundado pelo ambiente primitivo florestal. O relato e a discussão dos resultados obtidos constituem objeto do presente trabalho.

\section{LOCALIDADE ESTUDADA}

Para sediar estas observaçōes, foi escolhida a Fazenda Folha Larga, já anteriormente descrita, em seus aspectos gerais. As características correspondem a de local com atividade agropecuária em pequena escala, situado na margem da rodovia que liga as cidades de Pariquera-Açu e Cananéia. A abertura do terreno se deu em mata da planície Quaternária, que constitui revestimento vegetal primitivo tipicamente encontrado nessa regiāo meridional do Brasil (Klein ${ }^{12}$, 1984; Forattini e col. ${ }^{4}, 1986$ ). Sendo adjacente à floresta, apresenta áreas utilizadas para cultivo e pastagem, além de outras, temporariamente abandonadas, onde se observa processo de sucessão secundária. Todavia, tais feiçס̄es são freqüentemente modificadas e, quando do período de tempo em que foram feitas as presentes pesquisas, $o$ aspecto foi o representado pela Fig. Á extensão máxima do terreno aberto é de $540 \mathrm{~m}$, contados a partir da margem da rodovia, e nele encontravam-se instaladas três habitaçōes, uma delas contando com maior número de abrigos peridomiciliares destinados a animais domésticos. É de 6 ha a dimensão da área total da pastagem ali existente.

\section{MATERIAL E MÉTODO}

No período compreendido de julho de 1987 a agosto de 1988, foram coletados culicídeos adultos mediante a utilização da técnica de aspiração, como foi anteriormente descrita (Forattini e col.5, 1987). As pesquisas foram levadas a efeito nas seguintes situaçōes:

a) área de pastagem.

b) faixa de floresta, com cerca de $50 \mathrm{~m}$ de largura, contados a partir da margem.

c) peridomicinio.

Como se encontra assinalado na Fig., as áreas de mata foram designadas como A, B e C. As duas primeiras estão adjacentes ao terreno aberto e a terceira está situada no lado oposto da referida

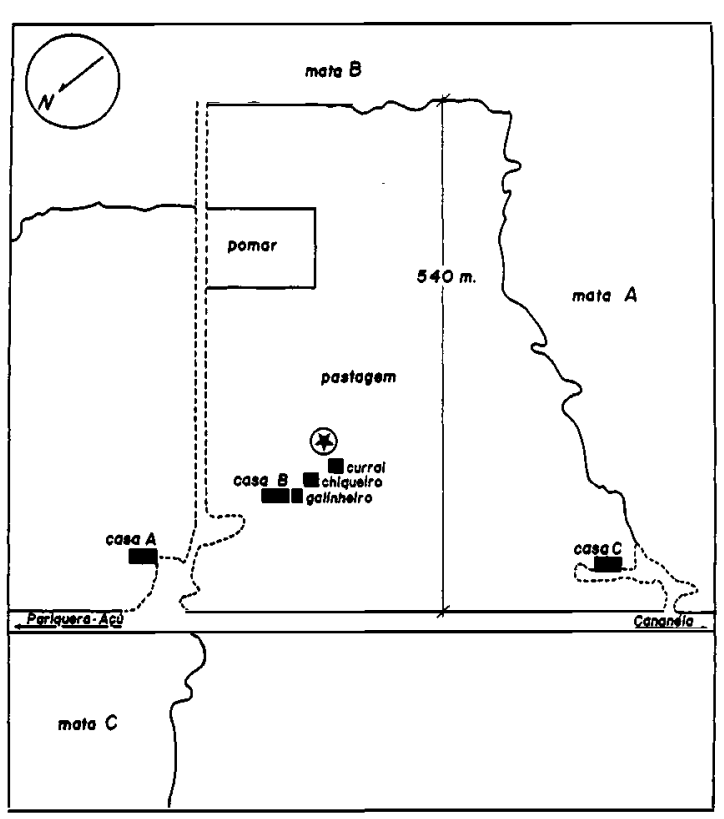

(4) estábulo com eqüino

Fig. Representação esquemática da área de estudo na Fazenda Folha Larga, Município de Cananéia, com a localizaçăo das casas e do estábulo com equüino, em relação as matas $A, B$ e $C$.

rodovia. Por razð̄es operacionais, as coletas no ambiente domiciliar limitaram-se ao peridomicílio das três casas existentes, e designadas de A, B e C, tendo sido pesquisados todos os possíveis abrigos. Estes incluíram um galinheiro, um chiqueiro e um curral bovino.

A coleta de adultos com o emprego de isca humana foi realizada na margem da mata $B$ e no peridomicilio da casa $B$, de maneira ininterrupta das $17: 00$ às $20: 00 \mathrm{~h}$. A regularidade de sua execução obedeceu a ritmo que impedisse a ocorrência de simultaneidade com a coleta de aspiração. Para isso, estabeleceu-se intervalo mínimo de 10 dias entre as duas, objetivando assim minimizar a influência desta no rendimento daquela.

Seguindo o método de Boreham e Port ${ }^{2}$ (1982), que objetiva evitar a manipulação de espécimens durante a marcação, procedeu-se à introdução local de um hospedeiro não existente na área em estudo, representado por um eqüino cujo sangue, uma vez identificado em mosquitos coletados nas diferentes situaçōes, desempenhou o papel de marcador. Em sendo assim, o animal foi confinado em estábulo, adrede preparado, durante os cinco dias que precederam a coleta na área (Fig.). Esta, por sua vez, foi executada mensalmente e abrangeu todos os supracitados ecótopos, no horário das 7:00 às 10:00 h. Simultaneamente à coleta, foi feito censo de todos os animais presentes nos dias em que ela ocorreu, bem como das pessoas que nessa ocasião pernoitaram nas três casas. 
TABELA 1

Identificação de fontes de repasto sangǘneo em representantes de Culicidae

\begin{tabular}{|c|c|c|c|c|c|c|c|}
\hline \multicolumn{8}{|c|}{ Fontes } \\
\hline Espécie & Ave & Bovino & Canídeo & Eqüino & Homem & Mista & Total \\
\hline $\begin{array}{l}\text { Aedes scapularis } \\
\text { Grupo serratus } \\
\text { Anopheles intermedius } \\
\text { Anopheles (Anopheles) sp. } \\
\text { Coquillettidia chrysonotum } \\
\text { Cq. oenezuelensis } \\
\text { Coquillettidia sp. } \\
\text { Culex mallis } \\
\text { Cx. nigripalpus } \\
\text { Cx. quinquefasciatus } \\
\text { Cx. (Culex) sp. } \\
\text { Cx. inadmirabilis } \\
\text { Cx. sacchettae } \\
\text { Cx. (Melanoconion) sp. } \\
\text { Psorophora ciliata } \\
\text { Ps. cingulata } \\
\text { Ps. confinnis } \\
\text { Ps. ferox } \\
\text { Uranotaenia geométrica }\end{array}$ & $\begin{array}{l}1 \\
3 \\
- \\
- \\
- \\
- \\
\overline{2} \\
3 \\
1 \\
\overline{1} \\
3 \\
- \\
- \\
\overline{1} \\
-\end{array}$ & $\begin{array}{r}45 \\
1 \\
3 \\
1 \\
3 \\
16 \\
2 \\
\overline{1} \\
- \\
\overline{8} \\
2 \\
3 \\
2 \\
1 \\
5 \\
1 \\
-\end{array}$ & $\begin{array}{l}- \\
\overline{-} \\
\overline{-} \\
\overline{-} \\
\overline{-} \\
\overline{1} \\
\overline{-} \\
\overline{-} \\
\overline{1} \\
\overline{-} \\
\overline{-} \\
-\end{array}$ & $\begin{array}{l}2 \\
1 \\
-1 \\
1 \\
5 \\
1 \\
- \\
- \\
- \\
\overline{1} \\
- \\
- \\
- \\
\overline{1} \\
\overline{1}\end{array}$ & $\begin{array}{l}1 \\
\overline{-} \\
\overline{-} \\
\overline{-} \\
\overline{1} \\
\overline{-} \\
\overline{-} \\
\overline{-} \\
\overline{1} \\
\overline{1} \\
\overline{6} \\
-\end{array}$ & $\begin{array}{c}2 \text { (be;ae) } \\
- \\
- \\
\overline{-} \\
1 \text { (be) } \\
\overline{-} \\
\overline{-} \\
1 \text { (bh) } \\
- \\
\overline{-} \\
\overline{-} \\
- \\
- \\
- \\
- \\
-\end{array}$ & $\begin{array}{r}51 \\
5 \\
3 \\
2 \\
5 \\
21 \\
3 \\
1 \\
3 \\
5 \\
1 \\
9 \\
3 \\
7 \\
3 \\
2 \\
6 \\
8 \\
1\end{array}$ \\
\hline Total & 15 & 94 & 2 & 14 & 10 & 4 & 139 \\
\hline
\end{tabular}

ae - ave + eqüino

be - bovino + eqüino

bh - bovino +homen

Considerando o conjunto representado pela casa B, o seu peridomicilio e o estábulo com eqüino, procedeu-se à medida das distâncias em relação às três áreas de mata supramencionadas. Os resultados, em linha reta, foram os seguintes:

$\begin{array}{cc}\text { mata } & \text { metros } \\ \text { A } & 345 \\ \text { B } & 407 \\ \text { C } & 160\end{array}$

Para a identificação do sangue ingerido foi utilizado o método do capilar, com o emprego de anti-soros para ave, bovino, canídeo, equiino e homem. Os índices alimentares ("feeding index") para o homem, em relação aos outros hospedeiros testados, foram calculados de acordo com a conceituação de Kay e col. ${ }^{11}$ (1979). Para isso, estimou-se o número de repastos esperados, pela contagem das populaçð̃es ali existentes. Como as capturas foram realizadas ao longo do período de 12 meses, os contingentes calculados representam a população média do período, o que pôde ser obtido mediante os referidos censos mensais.

\section{RESULTADOS}

Foi coletado o total de 31.133 mosquitos adultos, dos quais 29.820 fêmeas e 1.313 machos. A distribuição especifica dos espécimens femininos, por ordem de freqüência, foi a seguinte:

\section{Espécie}

Anopheles cruzii

An. bellator

Culex sacchettae

Aedes scapularis

Grupo serratus*

Psorophora ferox

Outras

Total

$\mathrm{N}$
19.487
4.708
92
761
363
240
3.337
29.820

$\%$

65,3

15,8

3,1

2,6

1,2

0,8

$\underline{11,2}$

100,0

* Inclui Ae. hastatus, Ae. nubilus, Ae. oligopistus e Ae. Serratus

Aspiração - Foram obtidos 4.030 exemplares dos quais 774 eram fêmeas das espécies mais comuns, assim distribuídas:

\begin{tabular}{lr}
\multicolumn{1}{c}{ Espécie } & N \\
Aedes scapuralis & 285 \\
Grupo serratus & 289 \\
Culex sacchettae & 50 \\
Psorophora ferox & $\underline{150}$ \\
Total & 774
\end{tabular}

Os demais repartiram-se por 57 espécies ou grupos, de comparecimento pouco expressivo. Além disso, assinalou-se a total ausência de Anopheles bellator e $A n$. cruzii no material coletado. 
No ambiente do peridomićlio foram obtidos 231 mosquitos de ambos os sexos, 78 dos quais representantes de espécies aqui focalizadas, as quais, juntamente com vários outros representantes de Culex (Melanoconion), se distribuíram da maneira seguinte:

\begin{tabular}{lrr}
\multicolumn{1}{c}{ Espécies (s) } & $\mathrm{N}$ \\
Aedes scapularis & 29 \\
Aedes serratus & 3 \\
Culex sacchettae & 4 \\
Cx. (Melanoconion) sp. & & 42 \\
$\quad$ Cx. inadmirabilis & 16 & \\
Cx. misionensis & 1 & \\
Cx. oedipus & 1 & \\
Cx. pilosus & 1 & \\
Cx. zeteki & 1 & \\
Cx. (Mel.) sp. & $\underline{2}$ & $\overline{78}$ \\
Total & 42 &
\end{tabular}

Os restantes das espécimens, ali coletadas, repartiram-se entre os gêneros Coquillettidia, Psorophora, além de outros.

Identificação de fontes sangüíneas - Com a técnica da aspiraçãa foram conseguidos 380 mosquitos engurgitados, 224 dos quais submetidos ao teste de precipitina para diagnóstico do sangue ingerido. Os 139 resultados positivos obtidos acham-se expostos na Tabela 1. No que concerne a Aedes scapularis, os resultados positivos, em relação às populaçżes médias de hospedeiros existentes, e estimadas mediante a realização de 12 censos ao longo do período de observaçðes, foram as seguintes:

Fonte População média Reações positivas

$\begin{array}{lcc}\text { Ave } & 73,42 & 2^{*} \\ \text { Bovino } & 22,67 & 46^{*} \\ \text { Eqüino } & 1,0 & 4^{* *} \\ \text { Homem } & 15,75 & 1 \\ & \\ \text { inclui uma reaçăo mista com eqüino } \\ \text { inclui uma reação mista com bovino e uma com ave. }\end{array}$

Com tais dados pôde-se calcular os índices alimentares ("feeding index") para cada grupo de hospedeiros, em relação a cada um dos demais, como consta na Tabela 2. Tais resultados indicaram que, para essas quatro categorias de fontes sangüíneas, o culicídeo manifestou maior preferência para eqüino, seguida pela escolha de bovino, homem e ave, nessa ordem.

Isca humana - Os resultados das coletas com a utilização de isca humana, levadas a efeito na margem da mata e no ambiente peridomiciliar, podem ser vistos na Tabela 3. Os dados ali expostos, transformados em médias de William, revelaram franca tendência por parte das quatro espécies ali focalizadas, de freqüentarem o ambiente humano. Nesse particular, destacou-se Anopheles
TABELA 2

f́ndices alimentares para Aedes scapularis, em relaçăo a algumas fontes sangüíneas*.

\begin{tabular}{lrccc}
\hline Fontes & Ave & Bovino & Eqüino & Homem \\
\hline Ave & 1,00 & 0,01 & 0,01 & 0,43 \\
Bovino & 74,49 & 1,00 & 0,51 & 32,25 \\
Eqüino & 146,84 & 1,97 & 1,00 & 63,0 \\
Homem & 2,33 & 0,03 & 0,02 & 1,00 \\
\hline
\end{tabular}

- Indice alimentar ("feeding index") da fonte mencionada na linha, em relação à fonte mencionada na coluna.

cruzii que, em alguns meses do periodo estival chuvoso, como janeiro e fevereiro, chegou a se apresentar com médias de até quase 240,0 nesse ambiente artificial humano.

Mobilidade pós-prandial - A utilização de um único hospedeiro para obter informaçōes sobre a possível movimentação de mosquitos, após o repasto sanguíneo, possibilitou a obtenção de alguns dados. Assim procedendo, foram obtidas 17 reaç̋es positivas para sangue de eqüino, como constam da Tabela 1. Sua distribuição pelos locais de coleta, de acordo com as distâncias em relação ao estábulo onde se manteve confinado o hospedeiro eqüino (Fig. ), foi a seguinte:

$\begin{array}{lccc}\text { Espécie } & \text { Local } & \begin{array}{c}\text { Distância } \\ \text { (ms) }\end{array} & \text { N } \\ \text { Aedes scapuralis } & \text { mata A } & 345 & 1 \\ & \text { mata B } & 407 & 1 \\ \text { Uranotaenia geométrica } & \text { mata C } & 160 & 2 \\ & \text { mata A } & 345 & 1\end{array}$

No ambiente peridomiciliar da casa $B$, onde esteve situado o estábulo supracitado (Fig. ), os casos positivos encontrados para esse hospedeiro foram os seguintes:

$$
\text { Espécie N }
$$

$\begin{array}{ll}\text { grupo serratus } & 1 \\ \text { Anopheles (Anopheles) sp. } & 1 \\ \text { Coquillettidia chrysonotum } & 2 \\ \text { Cq. venezuelensis } & 5 \\ \text { Coquillettidia sp. } & 1 \\ \text { Culex inadmirabilis } & 1 \\ \text { Psorophora confinnis } & 1\end{array}$

- Total

Pôde-se assim observar apreciável movimentação pós-prandial, por parte de espécimens de Aedes scapularis, que chegaram a percorrer distâncias ponderáveis após a realização do repasto sanguíneo. Em contraposição, nenhum exemplar de Anopheles bellator ou de An. cruzii foi co- 
TABELA 3

Resultados das coletas com isca humana, realizadas na margem da mata e no peridomicilio, no período de julho de 1987 a agosto de 1988, na Fazenda Folha Larga, Município de Cananéia.*

\begin{tabular}{|c|c|c|c|c|c|c|c|c|c|c|c|c|}
\hline \multirow{3}{*}{$\begin{array}{c}\text { Local e } \\
\text { Especies }\end{array}$} & \multicolumn{12}{|c|}{ Meses } \\
\hline & \multicolumn{2}{|c|}{ Janeiro } & \multicolumn{2}{|c|}{ Fevereiro } & \multicolumn{2}{|c|}{ Março } & \multicolumn{2}{|c|}{ Abril } & \multicolumn{2}{|c|}{ Maio } & \multicolumn{2}{|c|}{ Junho } \\
\hline & n & $\mathrm{mW}$ & $\mathrm{n}$ & mW & $\mathrm{n}$ & $\mathrm{mW}$ & $\mathrm{n}$ & $\mathrm{mW}$ & $\mathbf{n}$ & $\mathrm{mW}$ & $\mathrm{n}$ & $\mathrm{mW}$ \\
\hline $\begin{array}{l}\text { Margem da Mata } \\
\text { Aedes scapularis } \\
\text { Anopheles bellator } \\
\text { Aropheles cruzii } \\
\text { Culex sacchettae }\end{array}$ & $\begin{array}{r}- \\
11\end{array}$ & $\overline{1,3}$ & $\begin{array}{r}2 \\
215 \\
1.532 \\
4\end{array}$ & $\begin{array}{r}0,3 \\
10,6 \\
50,9 \\
0,6\end{array}$ & $\begin{array}{r}41 \\
596 \\
2.072 \\
143\end{array}$ & $\begin{array}{r}2,4 \\
27,9 \\
116,0 \\
7,8\end{array}$ & $\begin{array}{r}8 \\
183 \\
1.008 \\
75\end{array}$ & $\begin{array}{r}0,9 \\
17,7 \\
94,7 \\
7,2\end{array}$ & $\begin{array}{r}7 \\
146 \\
695 \\
20\end{array}$ & $\begin{array}{r}0,7 \\
12,1 \\
69,6 \\
2,0\end{array}$ & $\begin{array}{r}13 \\
52 \\
312 \\
7\end{array}$ & $\begin{array}{r}0,9 \\
4,1 \\
23,8 \\
0,4\end{array}$ \\
\hline Total & 11 & 1,3 & 1.753 & 62,4 & 2.852 & 154,1 & 1.274 & 120,5 & 868 & 84,4 & 384 & 29,2 \\
\hline $\begin{array}{l}\text { Peridomicllio } \\
\text { Aedes scapularis } \\
\text { Anopheles bellator } \\
\text { Anopheles cruzii } \\
\text { Culex sacchettae }\end{array}$ & $\begin{array}{r}76 \\
495 \\
2.023 \\
16\end{array}$ & $\begin{array}{r}7,8 \\
55,6 \\
239,5 \\
1,4\end{array}$ & $\begin{array}{r}40 \\
592 \\
1.740 \\
8\end{array}$ & $\begin{array}{r}4,9 \\
42,6 \\
90,5 \\
0,9\end{array}$ & $\begin{array}{r}79 \\
258 \\
411 \\
83\end{array}$ & $\begin{array}{r}5,9 \\
13,4 \\
28,5 \\
5,2\end{array}$ & $\begin{array}{l}26 \\
44 \\
30 \\
15\end{array}$ & $\begin{array}{l}2,8 \\
4,3 \\
3,7 \\
1,4\end{array}$ & $\begin{array}{r}16 \\
7 \\
7 \\
7\end{array}$ & $\begin{array}{l}1,5 \\
0,7 \\
0,7 \\
0,7\end{array}$ & $\begin{array}{r}8 \\
9 \\
25 \\
-\end{array}$ & $\begin{array}{r}1,0 \\
1,1 \\
2,3 \\
-\end{array}$ \\
\hline Total & 2.610 & 304,3 & 2.380 & 138,9 & 831 & 53,0 & 115 & 12,2 & 37 & 3,6 & 42 & 4,4 \\
\hline
\end{tabular}

\begin{tabular}{|c|c|c|c|c|c|c|c|c|c|c|c|c|c|c|}
\hline \multirow{3}{*}{$\begin{array}{l}\text { Local e } \\
\text { especies }\end{array}$} & \multicolumn{14}{|c|}{ Meses } \\
\hline & \multicolumn{2}{|c|}{ Julho } & \multicolumn{2}{|c|}{ Agosto } & \multicolumn{2}{|c|}{ Setembro } & \multicolumn{2}{|c|}{ Outubro } & \multicolumn{2}{|c|}{ Novembro } & \multicolumn{2}{|c|}{ Dezembro } & \multicolumn{2}{|c|}{ Total } \\
\hline & $\mathbf{n}$ & $\mathrm{mW}$ & $\mathbf{n}$ & $\mathrm{mW}$ & $\mathbf{n}$ & $\mathrm{mW}$ & $\mathrm{n}$ & $\mathrm{mW}$ & $\mathrm{n}$ & $\mathrm{mW}$ & $\mathrm{n}$ & $\mathrm{mW}$ & $\mathbf{n}$ & $\mathbf{m W}$ \\
\hline $\begin{array}{l}\text { Margem da Mata } \\
\text { Aedes scapularis } \\
\text { Anopheles bellator } \\
\text { Anopheles cruzii } \\
\text { Culex sacchettae }\end{array}$ & $\begin{array}{r}9 \\
116 \\
534 \\
10\end{array}$ & $\begin{array}{r}0,7 \\
11,4 \\
61,0 \\
0,6\end{array}$ & $\begin{array}{r}6 \\
219 \\
742 \\
36\end{array}$ & $\begin{array}{r}0,4 \\
17,9 \\
57,2 \\
2,2\end{array}$ & $\begin{array}{r}2 \\
228 \\
1.184 \\
152\end{array}$ & $\begin{array}{r}0,2 \\
22,6 \\
115,0 \\
11,2\end{array}$ & $\begin{array}{r}9 \\
385 \\
3.257 \\
40\end{array}$ & $\begin{array}{r}0,8 \\
36,9 \\
311,1 \\
3,7\end{array}$ & $\begin{array}{r}8 \\
432 \\
1.370 \\
12\end{array}$ & $\begin{array}{r}1,0 \\
46,0 \\
165,3 \\
1,1\end{array}$ & $\begin{array}{r}3 \\
374 \\
1.425 \\
17\end{array}$ & $\begin{array}{r}0,6 \\
42,5 \\
170,0 \\
1,1\end{array}$ & $\begin{array}{r}108 \\
2.946 \\
14.131 \\
527\end{array}$ & $\begin{array}{r}8,9 \\
249,7 \\
1.234,6 \\
39,2\end{array}$ \\
\hline Total & 669 & 73,7 & 1.003 & 77,7 & 1.566 & 149,0 & 3.691 & 352,5 & 1.822 & 213,4 & 1.819 & 214,2 & 17.712 & $1.532,4$ \\
\hline
\end{tabular}

Peridomicilio

\begin{tabular}{|c|c|c|c|c|c|c|c|c|c|c|c|c|c|c|}
\hline $\begin{array}{l}\text { Aedes scapularis } \\
\text { Anopheles bellator } \\
\text { Anopheles cruzii } \\
\text { Culex sacchettae }\end{array}$ & $\begin{array}{l}\mathbf{5} \\
\mathbf{1} \\
\mathbf{7} \\
\mathbf{3}\end{array}$ & $\begin{array}{l}0,9 \\
0,2 \\
1,6 \\
0,6\end{array}$ & $\begin{array}{l}15 \\
37 \\
41 \\
65\end{array}$ & $\begin{array}{l}1,3 \\
2,7 \\
2,4 \\
3,0\end{array}$ & $\begin{array}{r}3 \\
12 \\
121 \\
11\end{array}$ & $\begin{array}{l}0,3 \\
1,4 \\
3,9 \\
1,2\end{array}$ & $\begin{array}{r}44 \\
102 \\
500 \\
109\end{array}$ & $\begin{array}{r}2,4 \\
6,3 \\
29,9 \\
3,7\end{array}$ & $\begin{array}{r}18 \\
68 \\
37 \\
5\end{array}$ & $\begin{array}{l}1,5 \\
6,2 \\
4,3 \\
0,5\end{array}$ & $\begin{array}{r}38 \\
137 \\
414 \\
25\end{array}$ & $\begin{array}{r}2,4 \\
9,1 \\
15,4 \\
1,2\end{array}$ & $\begin{array}{r}368 \\
1.762 \\
5.356 \\
347\end{array}$ & $\begin{array}{r}32,7 \\
143,6 \\
422,7 \\
19,8\end{array}$ \\
\hline Total & 16 & 3,3 & 158 & 9,4 & 147 & 6,8 & 755 & 42,3 & 128 & 12,5 & 614 & 28,1 & 7.833 & 672,8 \\
\hline
\end{tabular}

- Para as especies mais freqüentes. mW - média de Williams.

letado pela aspiração, em que pese a elevada densidade no comparecimento às coletas com isca humana, tanto no peridomicilio como no meio ambiente natural representado pela mata circunjacente à área estudada.

Finalmente, e ainda focalizando o ambiente peridomiciliar, a identificação de sangue ingerido em espécimens engurgitados, ali encontrados, forneceu positividade quase que total para a fonte bovina, excluídos os casos supracitados, positivos para equiino. Tal resultado, muito provavelmente, encontra explicação na presença de curral para esses animais, nesse ambiente. Em tais circunstâncias, as espécies que forneceram resultados positivos para sangue de origem bovina foram Aedes scapularis (24), Coquillettidia venezuelensis (13), Culex inadmirabilis (8), Anopheles intermedius (3), Cq. chrysonotum (3), grupo serratus, An. (Ano- pheles) sp., Coquillettidia sp., Cx. sacchettae, $C x$. (Melanoconion) sp., Psorophora ciliata, Ps. cingulata e Ps. confinnis ( 1 cada). Alem disso, foram assinalados cinco espécimens de Culex quinquefasciatus nos quais foram identificadas as fontes ave (3), canídeo (1) e mista bovino/homem (1).

\section{DISCUSSÃO}

Os resultados obtidos em pesquisas anteriormente feitas nessa regiảo possibilitaram a identificação de algumas populações culicídeas apresentando tendência, em maior ou menor grau, para a domiciliação. Chegou-se a isso mediante a observação da freqüência ao ambiente domiciliar humano, bem como das preferências alimentares dirigidas para animais domésticos, quando não para o próprio homem (Forattini e col.5,6, , 1987, 1989). Com o precípuo objetivo de continuar os estudos so- 
bre esse tipo de comportamento, as presentes observações foram sediadas em área que tivessem contato direto com o ambiente natural primitivo, no caso a floresta da planície Quaternária do Sistema Tropical Atlântico. Isso possibilitou observar o comportamento de populaçōes de mosquitos silvestres em relação à área contígua, resultante das modificaçōes introduzidas pelo homem. Face aos supracitados resultados prévios, as atençбes dirigiram-se para Aedes scapularis, Anopheles bellator, An. cruzii e Culex sacchettae.

Há de se considerar que cerca de $87,0 \%$ das fêmeas coletadas foram representantes daquelas espécies, sobressaindo nitidamente $A n$. cruzii que, por si só, chegou a perfazer $65,3 \%$ do total. Isso veio confirmar o perfil já descrito para a região, 0 que, em relação à localidade estudada, permitiu deduzir pela inexistência de alteraçōes substanciais do ambiente que tivessem ocorrido desde aquela data (Forattini e col. ${ }^{4}, 1986$ ).

De maneira geral repetiu-se, por parte de Aedes scapularis, preferência para sugar animais de grande porte, como bovinos e eqüinos. Embora exista a influência por parte do nível de densidade dessas fontes no local, a estimativa do índice alimentar ("feeding index") parece confirmar essa preferência. E esse aspecto se reforça ao se levar em consideração a heterogeneidade local quanto às oportunidades para a escolha de fontes sangüineas (Garrett-Jones e col. ${ }^{8}, 1980$ ). Com efeito, os censos executados na área revelaram populações médias, não apenas para hospedeiros já mencionados e referindo-se a ave $(72,42)$, bovino $(22,67)$, homem $(15,75)$ e eqüino $(1,0)$, mas também as relativas a suíno $(9,08)$, canídeo $(5,45)$, coelho $(2,38)$ e gato $(1,0)$. Esse fato reveste-se de interesse ao se pretender avaliar a capacidade desse mosquito de ser atraído por animais domésticos existentes no ambiente humano.

No que concerne à capacidade de dispersão apetente, Ae. scapularis revelou indícios de apreciável movimentação pós-prandial, deslocando-se a distâncias de algumas centenas de metros a partir do local do repasto sanguíneo. Contudo, em boa parte, revelou também tendência a ali permanecer, no caso, o ambiente domiciliar humano, o que leva à suposição logica de que, ao mesmo tempo que tende a procurar abrigo no meio natural extradomiciliar, poderá encontrá-los no âmbito domiciliar do homem. Compare-se com o comportamento dos representantes do sub-gênero Kerteszia. Pelo que se observou, apesar de seu elevado comparecimento à isca humana, tanto Anopheles bellator como An. cruzii abandonam de imediato o local do repasto, provavelmente a procura de abrigos no ambiente natural que, no caso estudado, foi representado pela mata circunjacente. Assim, essas três espécies distinguiram-se localmente pelo aspecto de total mobilidade pós-prandial por parte dos anofelinos, enquanto aquele apresentou-a parcial- mente. Dessa maneira, o encontro em número relativamente pequeno, de espécimens engurgitadas no ambiente extradomiciliar, possivelmente sugere a ocorrência de dispersão sobre área consideravelmente extensa (Boreham e Port ${ }^{2}, 1982$ ).

A presença de sangue humano, em exemplares coletados por meio de aspiração, não poderá ser estritamente interpretado como possível movimentação pós-prandial a partir do ambiente domiciliar. Eis que os próprios capturadores poderiam ter servido de fontes alimentares, por ocasião das coletas.

Quanto à freqüência ao ambiente domiciliar, as coletas com o emprego de isca humana no peridomicílio e na mata mostraram que esse aspecto se mantém de maneira regular e paralelamente nos dois meios (Tabela 3). Tal feição assume interesse particular no caso de Culex sacchettae o qual, como espécie silvestre, parece assim demonstrar apreciável capacidade de exercer atividade no meio artificial, sugerindo claramente a possibilidade de domiciliação por parte também desse mosquito. No que concerne às demais espécies, os dados registrados, embora forneçam algumas informaçōes, não são de molde a possibilitar conclusőes mais solidas.

\section{CONCLUSÕES}

As observações que foram acima relatadas e comentadas, permitem as seguintes conclusőes:

1. A mobilidade pós-prandial de Aedes scapularis sugere atividade de dispersão do tipo apetente, em grau apreciável.

2. A freqüência ao meio humano, aqui representado pelo peridomicilio, se faz de maneira regular e paralela à atividade observada na mata, durante todo o período anual, por parte de Ae. scapularis, Anopheles bellator, An. cruzii e Culex sacchettae.

3. Dentre essas espécies, a primeira revelou maior número de indivíduos que permaneceram naquele ambiente após o repasto sanguíneo, o que permite concluir pela tendência à domiciliação.

4. Os anofelinos do subgênero Kerteszia, aqui representados por $A n$. bellator e $A n$. cruzii, năo mostraram propensão a permanecerem no ambiente domiciliar, motivo pelo qual sua capacidade de domiciliação deve ser considerada como praticamente inexistente.

5. Confirmaram-se as observações anteriores, a respeito da hematofagia de Ae. scapularis sobre animais domésticos, principalmente bovinos, para os quais parece possuir acentuada preferência.

6. A espécie $C x$. sacchettae revelou propensões 
à domiciliação, pelo menos face à freqüência domiciliar regular que se observou.

7. Em áreas próximas e adjacentes ao meio natural primitivo representado pela mata atlântica da planície, o ambiente construído pelo homem representa a criação de novos nichos ecológicos passíveis de serem ocupados por populações de culicídeos silvestres. Na localidade estudada estas foram representadas por Ae. scapularis e, embora em grau não tão evidente, também por $C x$. sacchettae..

FORATTINI, O. P. et al. [Frequency of occurrence in the human environment dispersal of Culicidae mosquitoes in an area adjacent to a primitive Atlantic plain forest]. Rev. Saúde públ., S. Paulo, 24: 101-7, 1990.

ABSTRACT: Results of peridomiciliar and forest environment mosquito catches are presented. By using the method single host, mosquitoes were identified according to blood-meal and thus their dispersal was observed. Evidence was obtained for movements of Aedes scapularis, due apparently were appetential flights, as well as its permanence in peridomiciliar resting places. Occurrence in domestic animal shelters was recorded for this species. Peridomiciliar human bait catches showed regular occurrence of Ae. scapularis, Anopheles bellator, An. cruzii and Culex sacchettae, but anophelines abandoned the human environment for the surrounding forest. Feeding index showed clearly a preference on the part of Ae. scapularis for bovine hosts. The peridomiciliar frequency of $C x$. sacchettae suggests a domiciliary tendency. The influence of large domestic animals in the human environment surrounded by primitive Atlantic plain forests, on the domiciliation of the sylvatic mosquitoes, is emphasized.

KEYWORDS: Mosquitoes. Aedes scapularis. Anopheles. Culex sacchettae. Population dispersion. Domiciliation. Food preferences.

\section{REFERENCIAS BIBLIOGRAFICAS}

1. BOREHAM, P. F. L.; LENAHAM, J. K.; BOULZAGUET, R.; STOREY, J.; ASHKAR, T. S.; NAMBIAR, R.; MATSUSHIMA, T. Studies on multiple feeding by $A n-$ opheles gambiae s.l. in a Sudan savanna area of north Nigeria. Trans. roy. Soc. trop. Med. Hyg., 73:418-23. 1979.

2. BOREHAM, P. F. L. \& PORT, G. R. The distribuition and movement of engorged females of Anopheles gambiae Giles (Diptera: Culicidae) in a Gambian village. Bull. ent. Res., 72:489-95, 1982.

3. DeFOLIART, G. R.; GRIMSTAD, P. R.; WATTS, D. M. Advances in mosquito-borne arbovirus/vector research. Ann. Rev. Entomol., 32:497-505, 1987.

4. FORATTINI, O. P.; GOMES, A. DE C.; NATAL, D.; SANTOS, J. L. F. Observações sobre atividades de mosquitos Culicidae em matas primitivas da planície e perfis epidemiológicos de vários ambientes no Vale do Ribeira, São Paulo, Brasil. Rev. Saúde públ., S. Paulo, 20:178203, 1986.

5. FORATTINI, O. P.; GOMES, A. DE C.; NATAL, D.; KAKITANI, I.; MARUCCI, D. Preferências alimentares de mosquitos Culicidae no Vale do Ribeira, São Paulo, Brasil. Rev. Saúde públ., S. Paulo, 21:171-87, 1987.

6. FORATTINI, O.P.; GOMES, A. DE C.; NATAL, D.; KAKITANI, I.; MARUCCI, D. Freqüência domiciliar e endofilia de mosquitos Culicidae no Vale do Ribeira, São Paulo, Brasil. Rev. Saúde públ., S. Paulo, 21:188-92, 1987.
7. FORATTINI, O. P.; GOMES, A. DE C.; NATAL, D.; KAKITANI, I; MARUCCI, D. Preferências alimentares e domiciliação de mosquitos Culicidae no Vale do Ribeira, São Paulo, Brasil, com especial referência a Aedes scapularis e a Culex (Melanoconion). Rev. Saúde públ., S. Paulo, 23:9-19, 1989.

8. GARRETT-JONES, C.; BOREHAM, P. F. L; PANT, C. P. Feeding habitats of anophelines (Diptera: Culicidae) in 1971-78, with reference to the human blood index: a review. Bull. ent. Res., 70:165-85, 1980.

9. GINSBERG, H. S. Dispersal pattems of Aedes sollicitans (Diptera:Culicidae) at the east end of Fire Island National Seashore, New York, USA. J. med. Ent., 23:146$55,1986$.

10. JOHNSON, C. G. Migration and dispersal of insects by flight. London, Methwen, 1969.

11. KAY, B. H.; BOREHAM, P. F. L; EDMAN, J. D. Application of the "feeding index" concept to studies of mosquito host-feeding patterns. Mosq. News, 39:68-72, 1979.

12. KLEDN, R. M. Aspectos dinâmicos da vegetação do sul do Brasil. Sellowia, 36:5-54, 1984.

Recebido para publicaçăo em S/12/1989 Aprovado para publicação em 23/1/1990 